\title{
Incerteza na Medição da Largura de Cordões de Solda
}

\author{
(Weld Bead Width Measurement Uncertainty)
}

\author{
Rosenda Valdés Arencibial ${ }^{1}$ José Ribamar dos Santos Ribeiro ${ }^{l}$ \\ ${ }^{1}$ Universidade Federal de Uberlândia, FEMEC, Av. João Naves de Ávila, 2121 - Bloco 1M, Uberlândia - MG, CEP 38400-902, \\ arvaldes@mecanica.ufu.br,jribamarribeiro@oi.com.br
}

\begin{abstract}
Resumo
O presente trabalho aborda a estimativa da incerteza associada à medição da largura do cordão de solda conforme recomendado pelo ISO GUM. A metodologia aplicada consistiu das seguintes etapas: identificação e estudo das variáveis que afetam a medição da largura do cordão, com paquímetro; adoção de um modelo matemático que relaciona as variáveis de entrada e de saída do sistema avaliado; planejamento e execução dos experimentos para o levantamento dos dados; estimativa da incerteza de medição e, finalmente, análise e discussão dos resultados. No final do trabalho foi possível concluir que a variabilidade associada às leituras, foi a fonte de maior contribuição para a incerteza final, e que a incerteza expandida atingiu o valor \pm 1,03 mm para 95,45\% de abrangência.
\end{abstract}

Palavras-chave: Incerteza, fator de abrangência e grau de liberdade efetivo.

\begin{abstract}
This paper addresses the estimation of the uncertainty associated to welding bead width measurement according to the ISO GUM recommendations. The applied methodology consisted of the following steps: identification and study of the variables that affect measurement of the bead width with a caliper; adoption of a mathematical model that relates the input and output variables of the evaluated system; planning and execution of experiments to gather data; estimation of measurement uncertainty and, finally, analysis and discussion of results. By the end of this work it was possible to conclude that the variability associated to the readings was the source of the largest contribution for the final uncertainty, and that the expanded uncertainty has reached the value $\pm 1,03 \mathrm{~mm}$ to $95,45 \%$ of coverage.
\end{abstract}

Key-words: Uncertainty, coverage factor and degrees of freedom.

\section{Introdução}

Todo resultado de medição é apenas uma estimativa do valor verdadeiro. Isto é devido à influência de diversos fatores que interferem no processo de medição, tais como variações associadas ao instrumento de medição, ao operador, às condições ambientes, entre outros. Por tal motivo, Institutos de Normalização Industrial de vários países sentiram a necessidade de indicar quantitativamente a qualidade do resultado de uma medição, ou seja, acrescentar ao resultado da medição uma declaração sobre a confiança associada a ele. Isso requer o estabelecimento de critérios para determinar o valor que representa a incerteza de medição. Esse valor pode ser considerado como um indicador do desempenho dos instrumentos de medição e da qualidade do valor medido [1].

Foi iniciada, então, uma busca sobre a melhor maneira de calcular a incerteza de medição e de introduzi-la nas normas de qualidade. Como conseqüência diversas organizações elaboraram documentos apresentando métodos para determiná-

(Recebido em 21/06/2009; Texto Final em 02/09/2009). la. No entanto, divergências em nomenclatura, conceitos básicos e métodos de avaliação não resultaram em um procedimento internacionalmente aceito.

Somente em 1993, com a publicação do "Guia para a Expressão da Incerteza em Medição" [2], foi possível contar com um documento que estabelece critérios e regras gerais para expressar e combinar as diferentes componentes da incerteza de medição. Assim sendo, ele tem sido adotado pela comunidade metrológica de diversos países. Segundo este documento, todo resultado de medição deve estar acompanhado da indicação quantitativa da qualidade do mesmo, de tal forma que aqueles que o utilizam possam avaliar sua confiabilidade. Sem essa indicação, os resultados de medição não podem ser comparados entre eles mesmos ou com valores de referência fornecidos em uma especificação ou norma.

Por outro lado, a referência [3] diz que todo resultado de medição deve estar relacionado a referências estabelecidas por padrões nacionais ou internacionais, através de uma cadeia contínua de comparações, todas tendo incertezas estabelecidas. Isto é, todo resultado de medição deve ser rastreável e para tanto, existe a obrigatoriedade da declaração da incerteza de medição.

Segundo a referência [4], na área de calibração, a incerteza é um conceito amplamente difundido e praticado pelos laboratórios. 
Entretanto, na área de ensaios, o cálculo da incerteza ainda não é uma prática totalmente adotada. Quando a incerteza não for declarada, o resultado da medição poderá tornar-se inválido.

Peloexpostoacima, este trabalho tem comoobjetivo apresentar uma metodologia para estimativa da incerteza associada à medição da largura do cordão de solda com paquímetro. Este mensurando é freqüentemente medido por pesquisadores da área de soldagem. Acredita-se que a declaração da incerteza aumentará o rigor científico dos trabalhos desenvolvidos e das publicações decorrentes dos mesmos.

\section{Erros de Medição e Incerteza}

A diferença entre o valor encontrado em uma medição e o valor verdadeiro é denominada erro de medição [5]. Em muitos processos de medição o valor verdadeiro é desconhecido, assim sendo, o erro de medição é dado pela diferença entre o resultado da medição e o valor obtido através da calibração.

De acordo com seu comportamento os erros de medição podem ser classificados em sistemáticos, aleatórios e histerese.

Erros sistemáticos são aqueles que permanecem constantes em grandeza e sinal ou variam, de acordo com uma lei definida, quando um número considerável de medições de um mesmo mensurando é efetuado sob as mesmas condições. Estes erros podem ser calculados e seus efeitos reduzidos a través de correções nos resultados finais da medição.

Os erros aleatórios são resultados de influências externas e internas, não controladas, que provocam o surgimento de erros não repetitivos. Em geral, diferem a cada leitura, podendo-se apenas ter noção dos seus limites. Tais erros estarão sempre presentes provocando variações nas medições sucessivas e somente podem ser avaliados estatisticamente.

Por sua vez a histerese é definida como sendo a diferença observada em cada posição de medição, quando o mensurando é avaliado nos sentidos crescente e decrescente da grandeza. Este erro pod e ser reduzido ou eliminado com um bom projeto, também, com ajuste e montagem adequadas dos elementos que compõem o instrumento de medição.

É sabido que os efeitos sistemáticos podem ser corrigidos sem grandes dificuldades, no entanto, a correção não é perfeita, permanecendo uma dúvida sobre o quão correto é o valor obtido em uma medição. Somando a esta dúvida aquela proveniente dos efeitos aleatórios, obtém-se o que se convencionou chamar de incerteza de medição.

A incerteza de medição pode ser definida como sendo o parâmetro, associado ao resultado de uma medição, que caracteriza a dispersão dos valores que poderiam ser razoavelmente atribuídos à grandeza [6]. Tal parâmetro pode ser o desvio padrão ou múltiplo dele ou a metade de um intervalo correspondente a um dado nível de confiança.

A palavra incerteza significa dúvida, assim sendo, a dúvida a cerca da validade do resultado de uma medição chama-se incerteza de medição. Isto é, a incerteza do resultado de uma medição reflete a falta de conhecimento exato do valor do mensurando.

$\mathrm{Na}$ incerteza de medição estão contidos os efeitos de todos os fatores que influenciam o resultado da medição, dentre eles:
$>$ Definição incompleta do mensurando;

$>$ Amostragem não representativa;

> Variações nas observações repetidas do mensurando sob condições aparentemente idênticas.

$>$ Desconhecimento dos efeitos das condições ambientais;

$>$ Erro de tendência pessoal na leitura de instrumentos analógicos;

$>$ Valores inexatos dos padrões de medição e materiais de referência;

$>$ Valores inexatos de constantes matemáticas e de outros parâmetros obtidos de fontes externas;

$>$ Aproximações e suposições ao método e procedimento de medição;

De forma geral, a estimativa da incerteza consta das seguintes etapas.

$>$ Identificar as variáveis que contribuem para a incerteza;

$>$ Escrever o modelo matemático;

$>$ Estimar a incerteza padrão associada a cada variável de entrada;

$>$ Estimar a incerteza padrão combinada;

$>$ Calcular o grau de liberdade efetivo;

$>$ Determinar o valor do fator de abrangência, k;

$>$ Estimar a incerteza expandida;

Apresentar os resultados.

\section{Incerteza na Medição da Largura do Cordão com Paquímetros}

O paquímetro é um instrumento de medição largamente utilizado na indústria para o controle dimensional de peças. Isto pode ser justificado pelo baixo custo e a possibilidade de medição de dimensões externas, internas, profundidades e ressaltos.

A medição da largura do cordão de solda é um processo que não exige equipamentos com alta exatidão, assim sendo, paquímetros com resolução de até $0,1 \mathrm{~mm}$ podem ser utilizados para este fim. Embora a utilização de paquímetros com melhor resolução permita diminuir a incerteza de medição, pois na maioria dos processos de medição com paquímetros, a resolução do mesmo constitui uma fonte de incerteza significativa.

A idéia de incorporar um nônio a uma régua graduada constituiu um grande passo na evolução dos instrumentos de medição, possibilitando efetuar medições com resolução de até $0,02 \mathrm{~mm}$. Porém, quando o cursor se desloca ao longo da régua estão presentes desvios angulares os quais influenciam o valor da medida e a incerteza de medição. Isto torna o paquímetro um instrumento bastante vulnerável ao erro de Abbé, surgindo sempre como candidato em potencial quando necessário apresentar um exemplo de instrumentos que não obedecem este princípio.

Os paquímetros não seguem as condições prescritas no princípio de Abbé. Existe uma folga causada pelo jogo do movimento do cursor bem como pela pressão da superfície de medição móvel contra a peça a ser medida. [7] recomenda que durante a medição de características externas com paquímetro, a peça objeto de medição deve entrar em contato com a superfície de medição do paquímetro o mais próximo possível da régua. Porém, quando se efetua a medição da largura do cordão de 
solda não é possível diminuir o braço de Abbé e o referido erro atinge seu máximo valor. Neste caso particular, a medição é feita utilizando-se as pontas dos bicos.

Uma análise visual de um cordão de solda revela que os limites do mesmo, nem sempre, estão bem definidos o que dificulta a medição. Assim sendo, de um ciclo de medição para outro a repetitividade das leituras é baixa, mesmo que o operador seja cuidadoso. Este fato deve ser considerado estimando a incerteza em diferentes pontos de medição distribuídos ao longo do cordão de solda.

As variações de temperatura ambiente, neste caso, não são consideradas. A justificativa está no fato de que os efeitos da dilatação térmica não são percebidos pelo paquímetro, pois sua resolução é limitada e, além disso, a largura do cordão assume valores pequenos.

De acordo com o exposto acima, o modelo para estimativa da incerteza associada à medição da largura do cordão de solda pode ser descrito pela Equação 1 .

$L \arg$ ura $=L_{P Q}+R_{P Q}+E_{\text {Abbe }}$

onde:

$>$ Largura: largura do cordão;

$>L_{P Q}:$ leituras indicadas pelo paquímetro;

$>R_{P Q}$ : resolução do paquímetro;

$>E_{\text {Abbè }}:$ Erro de Abbé.

A cada uma das variáveis presentes na Equação 1 está relacionada uma incerteza padrão. $\mathrm{O}$ documento [2] propõe duas formas para avaliação dessa incerteza, a saber: avaliação do tipo A e avaliação do tipo B.

A avaliação do tipo A é obtida estatisticamente a partir de medições ou observações repetidas de uma dada grandeza, assumindo uma distribuição normal ou outra qualquer. Enquanto que a avaliação tipo B da incerteza é feita a partir de um julgamento científico baseado em todas as informações relevantes disponíveis sobre o instrumento e o processo de medição [2]. O conjunto de informações pode incluir:

$>$ Dados de medições prévias;

$>$ Experiência ou conhecimento geral do comportamento e propriedades de materiais e instrumentos relevantes;

- Especificações do fabricante;

$>$ Dados fornecidos em certificados de calibrações ou outros certificados;

$>$ Incertezas relacionadas a dados de referências extraídos de manuais.

O uso adequado destas informações dependerá da experiência e do conhecimento geral, habilidade esta que pode ser adquirida, somente, com a prática.

Aplicando a lei de propagação de incertezas em (1) obtém-se a Equação 2. A referenciada lei de propagação de incerteza é baseada numa aproximação da série de Taylor de primeira ordem de $Y=f\left(x_{1}, x_{2}, \mathrm{~L} x_{N}\right)$.

$$
u_{c}^{2}(L \arg )=\left(\frac{\partial L \arg }{\partial L_{P Q}}\right)^{2}\left(u_{L_{P Q}}\right)^{2}+\left(\frac{\partial L \arg }{\partial R_{P Q}}\right)^{2}\left(u_{R_{P Q}}\right)^{2}+\left(\frac{\partial L \arg }{\partial E_{A b b e}}\right)^{2}\left(u_{E A b b e}\right)^{2}
$$

Neste caso, foi considerado que não existe correlação entre as variáveis.

A Equação 2 permite estimar a incerteza padrão combinada associada à medição da largura do cordão com probabilidade de abrangência de apenas 68,27 \% . Torna-se, então, necessário aumentar o intervalo para $95,45 \%$ de abrangência através do cálculo da incerteza expandida, Equação 3.

$U p=k \cdot u_{c}$

onde $u_{c}$ é a incerteza padrão combinada e $k>0$ é o fator de abrangência.

O fator $k$ está associado à distribuição de probabilidades dos valores obtidos na medição, que geralmente apresentam uma distribuição normal. É escolhido com base no nível de confiança requerido para o intervalo y-Up a y+Up.

Quando não for conveniente esta aproximação utiliza-se o teorema do valor central junto com a distribuição t-student para fornecer um fator de abrangência baseado no grau de liberdade efetivo da incerteza padrão da medição. O grau de liberdade efetivo $\left(v_{e f f}\right)$ é obtido através da fórmula de Welch-Satterthwaite,

Equação 4.

$$
v_{\text {efetivo }}=\frac{u_{c}^{4}(y)}{\sum\left(\frac{u_{i}^{4}(y)}{v_{i}}\right)}
$$

onde:

$>\mathrm{u}_{\mathrm{c}}$ : incerteza padrão combinada;

$>\mathrm{u}_{\mathrm{i}}$ : incerteza padrão associada a cada variável de entrada;

$>v_{\mathrm{i}}$ : grau de liberdade de cada distribuição.

\section{Experimentos, Resultados e Discussão}

Uma solda foi aplicada em um aço 1020 usando o processo MAG. Em seguida, a largura do cordão foi medida com um paquímetro analógico, de resolução $0,05 \mathrm{~mm}$, Figura 1. Tanto a solda quanto as medições foram conduzidas no laboratório de soldagem (LAPROSOLDA) da Faculdade de Engenharia Mecânica da Universidade Federal de Uberlândia.

Durante a medição nove pontos foram identificados ao longo do cordão e cinco ciclos de leituras foram tomados. A distância entre os pontos de medição foi predefinida visando à obtenção de uma amostra aleatória. Na Tabela 1, são apresentadas as indicações obtidas. 
Valdés, R. A., Ribeiro, J. R. S.

Tabela 1. Leituras (mm).

\begin{tabular}{c|c|c|c|c|c|c|c|c|c}
\hline & \multicolumn{10}{|c}{ Pontos de medição } \\
\hline Ciclo de medição & 1 & 2 & 3 & 4 & 5 & 6 & 7 & 8 & 9 \\
\hline 1 & 13,80 & 12,40 & 12,20 & 14,80 & 12,70 & 13,00 & 13,75 & 14,20 & 14,15 \\
\hline 2 & 13,80 & 12,25 & 11,90 & 15,00 & 12,50 & 14,10 & 14,15 & 14,00 & 14,15 \\
\hline 3 & 13,70 & 12,15 & 11,80 & 15,00 & 11,80 & 13,60 & 15,00 & 14,85 & 14,10 \\
\hline 4 & 13,80 & 12,30 & 11,60 & 14,30 & 12,65 & 13,55 & 14,80 & 14,00 & 14,25 \\
\hline 5 & 13,90 & 12,65 & 12,00 & 13,10 & 12,75 & 14,80 & 14,90 & 14,20 & 14,60 \\
\hline
\end{tabular}

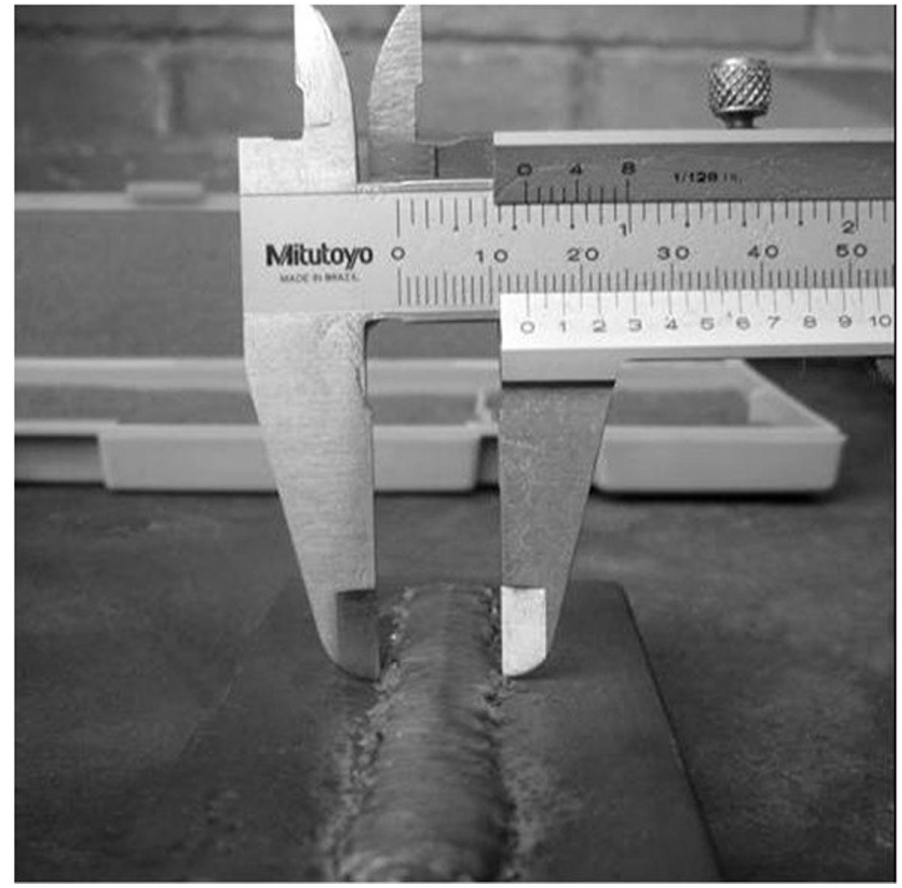

Figura 1. Cordão de solda durante a medição com paquímetro.

De posse dos dados foi calculada a incerteza de medição. Todas as etapas necessárias para a estimativa estão descritas de forma detalhada, a seguir.

\subsection{Identificação das variáveis que contribuem para a incerteza}

Conforme descrito anteriormente, as variáveis que contribuem para a incerteza na medição da largura do cordão de solda com paquímetro são:

Variabilidade das leituras indicadas pelo paquímetro;

Resolução do paquímetro;

Erro de Abbé.

\subsection{Adoção do modelo matemático}

O modelo matemático é dado pela Equação 1.

\subsection{Estimativa da incerteza padrão}

Nesta etapa foram calculadas as incertezas-padrão associadas a cada uma das variáveis de entrada.

\subsubsection{Incerteza padrão associada às leituras em cada ponto de medição.}

A incerteza padrão associada às leituras em cada ponto de medição pode ser estimada pela Equação 5 através de uma avaliação do Tipo A. A distribuição de probabilidades associada às leituras em cada ponto é a distribuição normal, com n-1 graus de liberdade.

$$
u\left(L_{m}\right)=\sqrt{\frac{s^{2}}{n}}
$$

a. Calcular a média aritmética considerando as leituras em cada ponto.

$$
\bar{x}=\frac{\sum_{i=1}^{1} x_{i}}{n}
$$

b. Calcular o desvio padrão considerando as leituras em cada ponto.

$$
s=\sqrt{\frac{\sum_{i=1}^{n}\left(x_{i}-\bar{x}\right)^{2}}{n-1}}
$$

A incerteza padrão associada à variabilidade das leituras, no ponto 1 , é de $0,03 \mathrm{~mm}$.

$$
u\left(L_{p q}\right)=\sqrt{\frac{s^{2}}{n}}=\sqrt{\frac{(0,0707 m m)^{2}}{5}}= \pm 0,0316 \mathrm{~mm}
$$

De forma análoga é estimada a incerteza padrão na totalidade dos pontos de medição.

Na Tabela 2, são mostrados os valores da média, desvio padrão e incerteza padrão encontrados em cada ponto de medição. Cabe ressaltar que os valores apresentados, na tabela, têm o número de algarismos significativos segundo a medição em análise, porém, em todas as etapas intermediárias para cálculo da incerteza foram consideradas quatro casas depois da vírgula. 
Tabela 2. Incertezas-padrão (u) em cada ponto, em (mm).

\begin{tabular}{l|l|l|l|l|l|l|l|l|l}
\hline & \multicolumn{10}{|c}{ Pontos } \\
\hline & 1 & 2 & 3 & 4 & 5 & 6 & 7 & 8 & 9 \\
\hline $\bar{x}$ & 13,80 & 12,35 & 11,90 & 14,44 & 12,48 & 13,81 & 14,52 & 14,25 & 14,25 \\
\hline $\mathrm{s}$ & $\pm 0,07$ & $\pm 0,19$ & $\pm 0,22$ & $\pm 0,80$ & $\pm 0,39$ & $\pm 0,68$ & $\pm 0,54$ & $\pm 0,35$ & $\pm 0,20$ \\
\hline $\mathrm{u}$ & $\pm 0,03$ & $\pm 0,08$ & $\pm 0,10$ & $\pm 0,36$ & $\pm 0,18$ & $\pm 0,30$ & $\pm 0,24$ & $\pm 0,16$ & $\pm 0,09$ \\
\hline
\end{tabular}

Desta análise pode-se concluir que a variabilidade das leituras em cada ponto de medição apresenta uma incerteza padrão, que varia entre 0,03 e $0,36 \mathrm{~mm}$. Esta fonte de incerteza sempre estará presente, podendo ter uma influência menor quando o operador for mais cuidadoso.

\subsubsection{Incerteza padrão associada à resolução do paquímetro.}

A incerteza padrão associada à resolução do paquímetro é do Tipo B e pode ser estimada segundo a Equação 8, assumindose uma distribuição de probabilidades retangular, com $\infty$ graus de liberdade. O paquímetro utilizado é do tipo analógico e tem resolução de $0,05 \mathrm{~mm}$.

$$
u\left(R_{p q}\right)=\frac{\operatorname{Re} \text { solução }}{\sqrt{3}}=\frac{0,05 \mathrm{~mm}}{\sqrt{3}}=0,0289 \mathrm{~mm}
$$

\subsubsection{Incerteza padrão associada ao erro de Abbé.}

O erro de Abbé está relacionado com o desvio de paralelismo entre as faces de medição para externos, também, com o esforço de medição. Este desvio é declarado no certificado de calibração do paquímetro e assume valores de $5 \mu \mathrm{m}$, aproximadamente. Assim sendo, a incerteza padrão associada ao erro de Abbé pode ser estimada pela Equação 9. A incerteza padrão associada ao erro de Abbé é do Tipo B com distribuição de probabilidades retangular e $\infty$ graus de liberdade.

$u\left(E_{A b b e}\right)=\frac{\text { Desvio de } p a r_{E x t}}{\sqrt{3}}=\frac{0,005 \mathrm{~mm}}{\sqrt{3}}=0,0029 \mathrm{~mm}$

\subsection{Estimativa da incerteza padrão combinada $\left(u_{c}\right)$}

Estimadas as incertezas-padrão de cada variável de influência, procede-se à combinação delas para cálculo da incerteza padrão combinada para cada um dos pontos de medição. A Tabela 3 mostra valores de incerteza, calculados através da Equação 2.

A incerteza padrão combinada associada à medição da largura do cordão de solda, no ponto 1 , é de $0,04 \mathrm{~mm}$ no ponto 1 para $68,27 \%$ de abrangência. Este valor pode ser representado graficamente como sendo um intervalo em torno da média.

Tabela 3. Incerteza padrão combinada em cada ponto, em mm.

\begin{tabular}{c|c|c|c|c|c|c|c|c}
\hline \multicolumn{1}{c}{ Pontos } \\
\hline 1 & 2 & 3 & 4 & 5 & 6 & 7 & 8 & 9 \\
\hline $\pm 0,04$ & $\pm 0,09$ & $\pm 0,10$ & $\pm 0,02$ & $\pm 0,18$ & $\pm 0,30$ & $\pm 0,24$ & $\pm 0,16$ & $\pm 0,10$ \\
\hline
\end{tabular}

\subsection{Estimativa da incerteza expandida (Up)}

A incerteza expandida, também deve ser calculada para cada um dos pontos de medição, através da Equação 3, pois os valores do grau de liberdade efetivo e de k são diferentes para cada um deles.

\subsection{Cálculo do grau de liberdade efetivo $\left(v_{\text {effetivo }}\right)$.}

O grau de liberdade efetivo é dado pela Equação 10.

$$
v_{\text {efetivo }}=\frac{u_{c}^{4}(y)}{\sum\left(\frac{u_{i}^{4}(y)}{v_{i}}\right)}
$$

Neste caso, para o ponto 1, tem-se:

$$
\begin{aligned}
& v_{\text {effitivo }}=\frac{u_{c}^{4}(y)}{\sum_{i=1}^{n}\left(\frac{u_{i}^{4}(y)}{v_{i}}\right)}=\frac{u_{c}^{4}(y)}{\sum_{i=1}^{3}\left(\frac{u_{i}^{4}(y)}{v_{i}}\right)}
\end{aligned}
$$

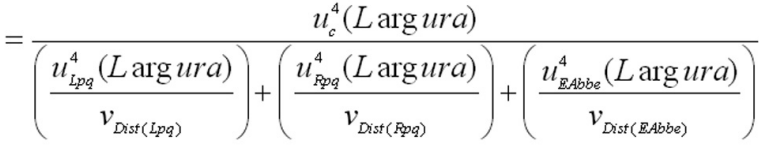

$$
\begin{aligned}
& =\frac{(0,0430 \mathrm{~mm})^{4}}{\frac{(0,0316 \mathrm{~mm})^{4}}{4}+\frac{(0,0288 \mathrm{~mm})^{4}}{\infty}+\frac{(0,0028 \mathrm{~mm})^{4}}{\infty}} \\
& =13,71
\end{aligned}
$$

portanto $\mathrm{k}=2,20$

$U p=k \cdot u_{c}=2,05 \cdot( \pm 0,0430 \mathrm{~mm})= \pm 0,0882 \mathrm{~mm}= \pm 0,09 \mathrm{~mm}$

\section{Forma padrão para apresentar o cálculo de incerteza}

Todas as informações referentes ao cálculo da incerteza devem ser apresentadas de forma resumida em uma tabela (Tabela 4).

A largura do cordão de solda, no ponto 1 , é de 13,80 $\pm 0,09$ $\mathrm{mm}$, para 95,45\% de abrangência e $\mathrm{k}=2,20$. Ela pode ser escrita como:

Largura do cordão $=13,80 \pm 0,98 \mathrm{~mm}$ 
Tabela 4. Incerteza associada à medição da largura do cordão no ponto 1.

\begin{tabular}{|c|c|c|c|c|c|}
\hline $\begin{array}{c}\text { Grandeza } \\
\mathrm{X}_{\mathrm{i}} \\
\end{array}$ & $\begin{array}{c}\text { Estimativa } x_{i} \\
(\mathrm{~mm})\end{array}$ & $\begin{array}{c}\text { Distribuição } \\
\text { de probabilidade }\end{array}$ & $\begin{array}{l}\text { Coeficiente de } \\
\text { sensibilidade }\end{array}$ & $\begin{array}{l}\text { Graus de } \\
\text { liberdade }\end{array}$ & $\begin{array}{l}\text { Incerteza padrão } \\
(\mathrm{mm})\end{array}$ \\
\hline $\mathrm{L}_{\mathrm{pq}}$ & 13,8000 & Normal & 1 & 4 & $\pm 0,0316$ \\
\hline $\mathrm{R}_{\mathrm{pq}}$ & 0,05 & Retangular & 1 & $\infty$ & $\pm 0,0289$ \\
\hline $\mathrm{E}_{\text {Abbe }}$ & 0,005 & Retangular & 1 & $\infty$ & $\pm 0,0029$ \\
\hline \multicolumn{5}{|c|}{ Incerteza padrão combinada $\left(u_{c}\right)$ em mm } & $\pm 0,04$ \\
\hline \multicolumn{5}{|c|}{ Grau de liberdade efetivo $\left(v_{e f f}\right)$} & 13,71 \\
\hline \multicolumn{5}{|c|}{ Fator de abrangência $(95,45 \%)$} & $\mathrm{k}=2,20$ \\
\hline \multicolumn{5}{|c|}{ Incerteza expandida (Up) em mm } & $\pm 0,09$ \\
\hline
\end{tabular}

Tabela 5. Incertezas-padrão combinada e expandida ( $\mathrm{mm})$.

\begin{tabular}{l|c|c|c|c|c|c|c|c|c}
\hline & \multicolumn{1}{l}{ Pontos } \\
& 1 & 2 & 3 & 4 & 5 & 6 & 7 & 8 & 9 \\
\hline $\bar{x}$ & 13,80 & 12,35 & 11,90 & 14,44 & 12,48 & 13,81 & 14,52 & 14,25 & 14,25 \\
\hline $\mathrm{S}$ & $\pm 0,04$ & $\pm 0,09$ & $\pm 0,10$ & $\pm 0,02$ & $\pm 0,18$ & $\pm 0,30$ & $\pm 0,24$ & $\pm 0,16$ & $\pm 0,10$ \\
\hline $\mathrm{U}$ & $\pm 0,03$ & $\pm 0,08$ & $\pm 0,10$ & $\pm 0,36$ & $\pm 0,17$ & $\pm 0,30$ & $\pm 0,24$ & $\pm 0,16$ & $\pm 0,09$ \\
\hline $\mathrm{Uc}$ & $\pm 0,04$ & $\pm 0,09$ & $\pm 0,10$ & $\pm 0,36$ & $\pm 0,18$ & $\pm 0,30$ & $\pm 0,24$ & $\pm 0,16$ & $\pm 0,10$ \\
\hline Vefetivo & 13,71 & 4,98 & 4,70 & 4,05 & 4,22 & 4,07 & 4,11 & 4,28 & 4,86 \\
\hline $\mathrm{K}$ & 2,20 & 2,65 & 2,7 & 2,87 & 2,8 & 2,87 & 2,85 & 2,82 & 2,72 \\
\hline $\mathrm{Up}$ & $\pm 0,10$ & $\pm 0,24$ & $\pm 0,28$ & $\pm 1,03$ & $\pm 0,50$ & $\pm 0,87$ & $\pm 0,70$ & $\pm 0,45$ & $\pm 0,26$ \\
\hline
\end{tabular}

\section{Cálculo da incerteza padrão combinada e expandida na totalidade dos pontos.}

De forma similar foi estimada a incerteza expandida na totalidade dos pontos de medição. Os resultados estão apresentados na Figura 2.

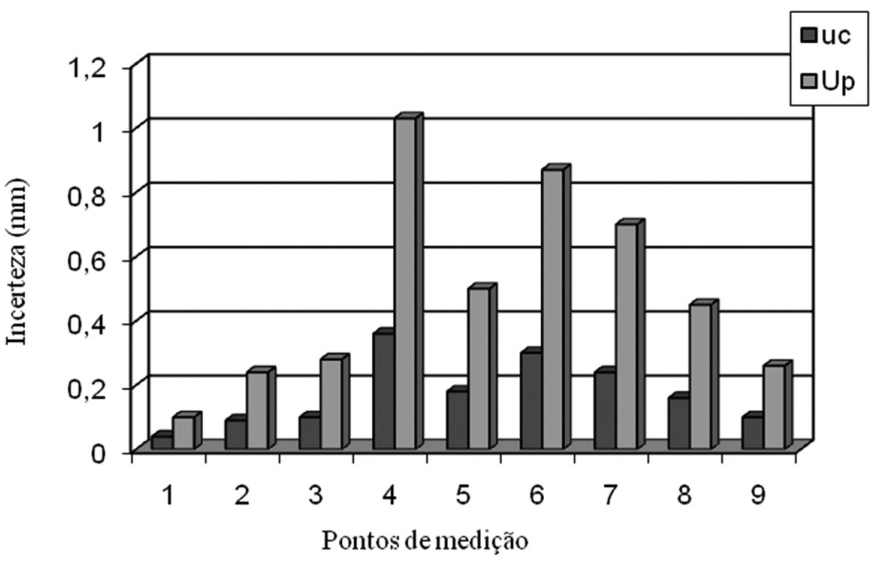

Figura 2. Incertezas padrão e expandida nos diferentes pontos de medição.
Na Tabela 5, são apresentados os valores da média e do desvio padrão e, também, os valores das incertezas padrão, padrão combinada e expandida.

Por fim, o cordão de solda apresenta uma largura média de $13,53 \pm 1,03 \mathrm{~mm}$ para uma probabilidade de abrangência de $95,45 \%$.

No resultado apresentado, a largura do cordão de solda é considerada como sendo a média das médias dos valores indicados pelo paquímetro, nos nove pontos de medição. Enquanto, que a incerteza expandida assume o máximo valor de incerteza encontrado, neste caso, no quarto ponto de medição.

\section{Conclusões}

Ao finalizar este trabalho podem ser apresentadas as seguintes conclusões:

a) Neste trabalho foi estimada a incerteza associada à medição, com paquímetro, da largura do cordão de solda, utilizando [2].

b) Os cálculos envolvidos para determinar a incerteza associada às medições são simples.

c) Observou-se que a variabilidade associada às leituras foi a fonte de maior contribuição para a incerteza final na medição da largura do cordão. As diferenças observadas entre os 
valores estimados, nos nove pontos de medição, podem ser atribuídas à indefinição dos limites do cordão e à habilidade do operador.

d) A resolução do paquímetro, também, contribuiu de forma significativa para a incerteza final. Esta parcela de contribuição pode ser minimizada utilizando um paquímetro com resolução melhor.

e) A incerteza padrão combinada associada à medição da largura do cordão de solda assumiu valores de até $\pm 0,36 \mathrm{~mm}$, para $68,27 \%$ de abrangência. Por sua vez, a incerteza expandida atingiu o valor $\pm 1,03 \mathrm{~mm}$ para $95,45 \%$.

\section{Referências}

[1] NIST TECNICAL NOTE 1297. Guide lines for evaluating and expressing the uncertainty of NIST measurement results. National Institute of standards and technology. 1994.

[2] ISO TAG 4/WG 3. Guide to the Expression of Uncertainty in Measurement, Geneva Switzerland, 1993.

[3] NBR ISO/IEC 17025. Requisitos gerais para competência de laboratórios de ensaio e calibração. Janeiro, 2001.

[4] JORNADA D.H. Calcular a incerteza em ensaios é realmente importante? Jornal da Metrologia. Ano 8 - Edição número 59. 1p. Março de 2007.

[5] INMETRO. Vocabulário Internacional de Termos Fundamentais e Gerais de Metrologia - VIM. 2007.

[6] INMETRO. Guia para Expressão da Incerteza de Medição, Rio de Janeiro, $3^{\text {a }}$ Edição Brasileira, 2003.

[7] ABNT NBR NM 216. Paquímetros e paquímetros de profundidade - Características construtivas e requisitos metrológicos 2000. 